\title{
Codes graphiques et place sociale de la langue occitane à travers son histoire
}

Graphic codes and the social place of the Occitan language throughout its history

\section{Hervé Lieutard}

\section{OpenEdition}

\section{Journals}

Édition électronique

URL : https://journals.openedition.org/lbl/456

DOI : $10.4000 / \mathrm{lbl} .456$

ISSN : 2727-9383

\section{Éditeur}

Université de Bretagne Occidentale - UBO

\section{Édition imprimée}

Date de publication : 30 décembre 2019

Pagination : 9-28

ISBN : 979-10-92331-49-3

ISSN : 1270-2412

\section{Référence électronique}

Hervé Lieutard, "Codes graphiques et place sociale de la langue occitane à travers son histoire ", La

Bretagne Linguistique [En ligne], 23 | 2019, mis en ligne le 01 mai 2020, consulté le 04 mai 2021. URL http://journals.openedition.org//bl/456 ; DOl : https://doi.org/10.4000/lbl.456

\section{cc) (†)}

La Bretagne Linguistique est mise à disposition selon les termes de la Licence Creative Commons Attribution 4.0 International. 


\title{
Codes graphiques et place sociale de la langue occitane à travers son histoire
}

\author{
HERVÉ LIEUTARD
}

T 'élaboration d'un système graphique original qui caractérise l'occitan _au Moyen Âge, son délabrement spectaculaire au XVI ${ }^{\mathrm{e}}$ siècle et sa laborieuse renaissance à partir du XVIII ${ }^{e}$ siècle sont les diverses facettes d'une même histoire, celle de la langue occitane soumise à des contraintes sociales et politiques internes ou externes qui varient dans le temps. Ainsi les changements graphiques au fil des siècles peuvent être perçus comme des témoins majeurs des bouleversements sociolinguistiques qui ont affecté cette langue tout au long de son histoire. Il ne s'agit pas ici de proposer une réflexion sur les pratiques graphiques actuelles ${ }^{1}$, mais de présenter quelques moments-clés de l'histoire de la langue occitane en lien avec ses usages graphiques, pour tenter de comprendre ce qu'ils nous disent de la place sociale de la langue à divers moments de son histoire, mais aussi pour mieux comprendre la difficile genèse des premières normes graphiques contemporaines, représentées par la norme félibréenne et la norme classique.

\footnotetext{
* Université Paul-Valéry Montpellier 3, LLACS EA 4582, F34000, Montpellier, France

1. Sur cette question précise, cf. James COSTA et Médéric GASQUET-CYRUS (dir.), Aspects idéologiques des débats linguistiques en Provence et ailleurs, Lengas [En ligne], $\mathrm{n}^{\circ} 72$ | 2012, mis en ligne le 25 octobre 2013, consulté le 29 novembre 2018. URL : http://journals.openedition.org/lengas/109; DOI : 10.4000/lengas.109.
} 


\section{L’émergence de la graphie classique au Moyen Âge}

Un faisceau de raisons a concouru au développement de l'écrit occitan au Moyen Âge dans de nombreux registres jusque-là réservés au latin, en particulier vers le milieu du XIII ${ }^{e}$ siècle. Le prestige des troubadours dont les productions sont dorénavant recueillies et compilées dans les divers chansonniers a sans aucun doute contribué à la valorisation de l'usage de l'occitan dans d'autres registres écrits juridiques, administratifs ou scientifiques - dans lequel il était jusqu'alors fortement concurrencé par le latin. Le développement de l'écrit occitan non-littéraire dans les registres pragmatiques au sein du Consulat de Montpellier (1204-1789) est représentatif du lien entre la place sociale de la langue occitane et ses possibilités de développement. Le passage du pouvoir des mains de l'aristocratie locale, les Guilhèm, à celles des représentants locaux de l'activité commerciale et économique de la ville, les consuls, s'accompagne de l'abandon progressif du latin dans les textes officiels montpelliérains. L'héritage majeur de ce gouvernement communal médiéval, basé sur un système de représentation par les métiers, est le manuscrit AA9 dit du Petit Thalamus ${ }^{2}$, conservé aux Archives municipales de Montpellier. Exécuté à partir des années 1320, il compile les autres livres du gouvernement consulaire, au nombre de sept, dont la rédaction en occitan a commencé un siècle plus tôt, dans la seconde moitié du XIII e siècle. La rédaction en occitan du Petit Thalamus s'arrêtant au $\mathrm{XV}^{\mathrm{e}}$ siècle pour reprendre en français au tout début du $\mathrm{XVI}^{\mathrm{e}}$ siècle, ce manuscrit offre donc une perspective intéressante sur trois siècles de pratique écrite de l'occitan.

En compilant et en réécrivant la mémoire officielle de Montpellier à partir des premiers manuscrits, le manuscrit AA9 apparaît comme la somme des pratiques régulatrices graphiques et des efforts de standardisation des générations précédentes. Un siècle après les premiers Thalami, vers 1340-1360, on voit effectivement se mettre en place une forme relativement homogène d'écriture de l'occitan par rapport aux choix graphiques hétérogènes qui caractérisaient les premiers manuscrits montpelliérains dont la graphie restait soumise aux contraintes de

2. L'ensemble des Thalami du Consulat a récemment fait l'objet d'une édition électronique (http://thalamus.huma-num.fr) dans le cadre d'un projet ANR ayant regroupé occitanistes, juristes et historiens de Montpellier. 
l'alphabet latin. Cette homogénéisation graphique est sans doute en partie liée à la "fonctionnarisation " des notaires ${ }^{3}$ au sein du consulat au $\mathrm{XIV}^{\mathrm{e}}$ siècle qui s'accompagne de nouvelles normes de travail, lesquelles contribuent à fixer et à stabiliser les pratiques graphiques. Ce modèle graphique performant est dorénavant capable de rendre compte de la langue occitane dans toute sa modernité. Il enregistre les évolutions phonétiques, morphologiques et morphosyntaxiques les plus récentes : les diphtongaisons sont notées, la morphologie verbale se rapproche peu à peu des formes modernes, les vestiges du système bi-casuel médiéval disparaissent, mais on voit surtout se fixer peu à peu un code graphique relativement stable et apparaître de nouvelles solutions pour rendre compte des phénomènes de palatalisation propres aux langues romanes, à l'instar des digrammes $<$ nh $>$ et $<\mathrm{lh}>$ pour la notation des consonnes palatales.

\begin{tabular}{|c|c|c|c|c|c|c|c|c|}
\hline & J339 & AA4 & $20807-809$ & naf 4337 & fr. 11795 & fr. 14507 & H119 & AA9 \\
\hline$<$ lh $>$ & 0 & 1 & 0 & 0 & 193 & 2 & 70 & 2038 \\
\hline$<$ nh $>$ & 10 & 33 & 8 & 24 & 300 & 12 & 64 & 2770 \\
\hline
\end{tabular}

Tableau 1 : Nombre d'occurrences des digrammes $<\mathrm{lh}\rangle$ et $<\mathrm{nh}\rangle$ dans les divers manuscrits du Petit Thalamus

Au-delà de cet aspect, on remarque également que le Petit Thalamus prend ses distances avec les réalisations orales populaires, ce que nous appelons aujourd'hui des réalisations dialectales, pour privilégier une forme d'occitan bien moins identifiable d'un point de vue diatopique. Par exemple, la forme de la diphtongaison conditionnée de [־] issue de ŏ latin n'est pas celle de Montpellier, qui ne connait aujourd'hui que des diphtongues en <uò> [uગ] comme dans nuòch' (> nŏctem, nuit), mais une forme que l'on pourrait qualifier de plus générale en $<$ uè> $[u \varepsilon]$. On trouve ainsi dans notre manuscrit AA9, 68 occurrences de nuèch contre 8 occurrences seulement de nuòch ${ }^{4}$, cette dernière forme étant quasi

3. Pierre CHASTAng, La ville, le gouvernement et l'écrit à Montpellier (XII'-XIVe siècle). Essai d'histoire sociale, Paris, Publications de la Sorbonne, 2013, p. 118-119.

4. Je donne ici les formes orthographiques modernes correspondant respectivement à nuoch, nuog et à nuech, nueg, nuegz dans le manuscrit. Il est intéressant de noter que ces formes en $<$ uo $>$ ne se retrouvent que sur une brève période vers le milieu du $\mathrm{XIV}^{\mathrm{e}}$ siècle. 
absente dans les dernières années de rédaction, comme en rend compte l'extrait ci-dessous de l'année 1426 alors que c'est aujourd'hui la seule forme orale en usage dans le languedocien oriental.

"Item, l'an desus, fonc diverses veguadas terratremol en esta vila e comensset en lo mes de mars et en aquest an meteys fonc tant granda terratremol en Cathaluonha a Pirpinia, a Girona et a Barssalona e per tot lo pajs d'entorn que totz los jorns la y era III e IIII veguadas e aytant la nueg, en tant que la gent de las cieutatz anavon la nueg dormir foras la vila per paor que los hostals non tonbesson;"

Cette année-là, il y eut à plusieurs reprises des tremblements de terre dans notre ville ; cela commença au mois de mars et, cette même année, il y eut un si grand tremblement de terre en Catalogne à Perpignan, à Gérone et à Barcelone, ainsi que dans tout le pays environnant, qu'il s'y en produisait tous les jours trois ou quatre fois, et autant la nuit, si bien que les gens des cités allaient dormir la nuit en dehors des villes de peur que les maisons ne tombent ;

La forme graphique <ue>, qui est aussi la première à apparaître dans les anciens Petits Thalami, renvoie probablement à une forme de variation diaphasique propre au registre écrit, que nous pourrions qualifier aujourd'hui de forme référentielle ou prestigieuse, alors que la forme <uo> correspond sans doute déjà à l'époque à un usage oral, propre à la pratique populaire du languedocien oriental. S'il est clair que l'on n'écrit pas l'occitan comme on le parle, il est légitime de se demander si ce registre écrit prestigieux a pu correspondre également à Montpellier à une variation diastratique des usages oraux qui aurait pris pour modèle la variété de la haute société montpelliéraine en rejetant les formes populaires.

En l'absence de réponse définitive à cette question, sur laquelle je reviens dans la partie suivante, on relève cependant un fait assez frappant : la tendance à l'harmonisation graphique entre le Petit Thalamus montpelliérain et les textes écrits à la même époque à Toulouse, en particulier dans le traité de grammaire et de rhétorique de 1356 connu sous le nom de Leys d'Amors ${ }^{5}$. Dans ce manuscrit, émanation du Consistori del Gay Saber, l'académie poétique officiellement soutenue

5. Joseph ANGLADE (éd.), Las Leys d'amors: manuscrit de l'Académie des Jeux floraux, Toulouse, Privat, 4 vol., 1919-1920. 
par le Capitoulat de Toulouse qui organise des concours poétiques en occitan pour perpétuer la tradition lyrique troubadouresque, il est frappant de voir que là aussi, on n'use pas forcément des formes orales en usage dans le languedocien de Toulouse. Si la notation de certaines réalisations populaires est réprouvée dans le registre littéraire ${ }^{6}$, il est plus surprenant de noter dans ce texte toulousain la présence de formes palatalisées en $<\mathrm{ch}>[\mathrm{t}]$, issues du groupe latin -CT- puisque le languedocien méridional ne connaît aujourd'hui que des formes en <-it> [-jt] comme par exemple nuèit (> nŏctem, nuit) dans la norme actuelle. Les Leys d'Amors utilisent donc des formes lexicales telles que "fag» (fait) et "nueg» ${ }^{7}$ (nuit), soit fach et nuèch dans la norme actuelle et la terminologie grammaticale fait usage de formes telles «del dig preterit perfag ${ }^{8}$ (dudit prétérit parfait), del dich preterit perfach dans la norme actuelle. Toutes ces formes palatalisées sont conformes à celles que l'on trouve majoritairement en occitan, mais pas dans la variété dialectale actuelle de Toulouse. Comme pour Montpellier, rien n'empêche de penser là aussi que ces formes référentielles écrites aient pu jouir un temps d'un prestige particulier et correspondu à un emploi effectif oral dans une partie de la haute société toulousaine.

Une chose est sûre, les usages littéraires à Toulouse comme les usages pragmatiques à Montpellier impliquent l'existence de registres distincts de la langue populaire et de ses variations dialectales. Au bout du compte, on observe une forme d'homogénéisation des pratiques graphiques au niveau régional et sans doute à une échelle plus large qui s'explique par un contexte social et politique relativement favorable pour l'occitan lui permettant de dépasser sa simple fonction de communication orale quotidienne ou de transmission populaire pour occuper ces nouvelles fonctions prestigieuses. Ainsi, les pratiques graphiques médiévales de l'occitan renvoient à la question de la norme. Si toute norme graphique repose sur la somme des pratiques régulatrices des générations précédentes, on retiendra également qu'elle émane d'un groupe socioculturel détenteur d'un niveau d'instruction

\footnotetext{
6. Notamment la transcription graphique du bêtacisme.

7. J. ANGLADE, op. cit., vol. I, p. 19.

8. Ibidem, vol. III, p. 161 et passim.
} 
élevé, d'une forme de pouvoir ou d'une institution dont le prestige social est suffisamment fort pour lui permettre d'en assurer l'usage et la diffusion. Si d'un côté, on voit clairement s'établir des normes graphiques régionales, partiellement détachées des usages populaires, les pratiques graphiques à Toulouse et à Montpellier semblent montrer qu'une relative stabilité institutionnelle, que ce soit dans l'exercice du pouvoir ou dans l'exercice littéraire, est une des conditions d'émergence et de stabilisation d'une norme écrite.

\section{Vers un code graphique exogène : diglossie interne et diglossie externe}

Même si la place sociale de l'occitan dans les registres écrits commence véritablement de changer dès le $\mathrm{XV}^{e}$ siècle, c'est au XVI siècle que l'on prend la véritable mesure du profond bouleversement qui s'est effectué en l'espace de quelques décennies. Trois textes toulousains anonymes $^{9}$, parus la même année, en 1555 , sont assez caractéristiques de ces bouleversements sociolinguistiques. Ultime manifestation du système graphique classique de l'occitan, même si la norme occitane endogène s'y trouve d'ores et déjà affaiblie corrélativement à la montée du prestige du français - visible dans l'orthographe ou le lexique -, ces textes font la preuve qu'il est encore possible de jouer sur le souvenir des codes graphiques de l'occitan médiéval, notamment à travers la parodie de l'occitan officiel et littéraire ${ }^{10}$ des siècles précédents par les femmes de Toulouse, même si par ailleurs ces textes annoncent déjà la veine burlesque et parodique dans lequel l'occitan se trouvera de plus en plus relégué ${ }^{11}$.

9. La Requeste faicte \& baillée par les Dames de la Ville de Tolose, Tolosa, Jacques Colomiès, 1555 ; Las Ordenansas \& coustumas del Libre Blanc observadas de tota ancianetat, compausadas per las sabias femnas de Tolosa. Et registradas en forma deguda per lor secretary, Tolosa, Jacques Colomiès, 1555 ; Las Nompareilhas Receptas per far las Femnas tindentas, rizentas, plasentas, polidas \& bellas, Tolose, Guyon Boudeville, 1555. 10. C'est le cas notamment dans Las Ordenansas et Coustumas del Libre blanc, op. cit., où les femmes de Toulouse, à l'instar des consuls montpelliérains du Petit Thalamus ou des capitouls de Toulouse, sont devenues les représentantes du pouvoir dans la ville. 11. Hervé LieUtARD, Patrick SAUZET, "D'une diglossie à l'autre : observations linguistiques et sociolinguistiques sur deux textes toulousains de 1555: Las 
Au Moyen Âge, l'écrit semble avoir réussi, du moins en partie, à dépasser la variation diatopique, mais pour y parvenir il fallait sans doute qu'existent des registres et de variations diastratiques. Dans les textes toulousains de 1555, le "bel lengatge» n'est plus à présent représenté que par la variété de la grande ville, celle de Toulouse, qui s'érige en modèle mais en exhibant quantités de traits dialectaux hyperlocalistes ${ }^{12}$, parfois jusqu'à la caricature. À l'instar du français qui, à la même époque, érige la variété d'Île de France en modèle littéraire, les auteurs de ces textes voient de la même façon dans le seul dialecte toulousain les germes du beau langage occitan, capable de rivaliser avec le français comme l'exprime le manifeste linguistique occitan intitulé Epistre envoyée aux Dames de Paris de La Requeste ${ }^{13}$. Dans ce même recueil, deux rondeaux parodiques tournent en dérision les dialectes gascon et "gavach", et semblent avoir pour seule fonction de mieux faire ressortir par contraste les qualités du beau langage toulousain ${ }^{14}$. Dans cette manifestation clairement mise en scène d'une diglossie interne, on relèvera par exemple l'absence de bêtacisme dans la bouche des Dames de Toulouse et la profusion de $<\mathrm{b}>$ dans les formes linguistiques gasconnes caricaturées ("Goardatz bous ara, esta bila se es bera ", Voyez si cette ville est belle). Sont également tournées en dérision les variétés occitanes septentrionales, à travers le Rondeau en "gavach», signé fictivement par "Andieta Pechayre» et caractérisé par une accumulation outrancière de formes palatalisées en <cha> caractéristiques de cette zone linguistique ("micha», "charsalada», "serchan», "chanson», "dicha», "chala», "ficha», "chada»). Il est intéressant de noter que la caricature des dialectes gascon et gavach se fait ici par le recours à la

Ordenansas e coustumas del libre blanc et Las nonpareilhas receptas", Autour des quenouilles, la parole des femmes (1450-1600), Courouau, Gardy, Koopmans (eds.), Turnhout, Brepols, 2010, p. 109-146.

12. Pour une étude détaillée de la langue et des aspects sociolinguistiques de ces textes, cf. H. Lieutard, P. SAUZET, op. cit.

13. Jean-François Courouau, Philippe GARDY, La Requeste faicte et baillée par les Dames de la Ville de Tolose, édition critique, Toulouse, Presses universitaires du Mirail, 2003, p. 99-100.

14. Hervé LieUtARD, "Quelques remarques sur la graphie de deux rondeaux hyperdialectaux, extraits de la Requeste (1555)», Lengas [En ligne], n $68 \mid 2010$, mis en ligne le 25 mars 2015, consulté le 15 janvier 2019. URL : http://journals. openedition.org/lengas/671; DOI : 10.4000/lengas.671. 
graphie exogène du français qui sert à rendre compte phonétiquement des formes orales dialectales tournées en dérision. Notons également au passage que si le $<\mathrm{ch}>$ permet de railler la prononciation de l'occitan le plus septentrional, il est aussi utilisé pour se moquer de la prononciation du français : "Car en parlan, semble que machetz fresas» ${ }^{15}$ (car en parlant, il semble que vous mâchiez des fèves), disent les Dames de Toulouse à celle de Paris.

De manière plus diffuse, et sans doute moins consciente que pour le rejet du bêtacisme qui peut encore résister comme un marqueur de la stratification sociale des usages, il reste toutefois encore possible de voir dans ces textes un autre indice des vestiges d'une stratification sociale des usages à travers la persistance de quelques formes palatalisées de -CT latin en -ch, à l'instar de celles déjà signalées ci-dessus au XIV siècle dans les Leys d'Amors et qui ont pu jouir un temps d'un prestige particulier alors qu'elles ont complètement disparu aujourd'hui du dialecte toulousain. Il est en effet difficile d'expliquer autrement que comme des formes de prestige les occurrences de "bruch» (bruit) dans La Requeste en face de "bruct» dans le même recueil ou de "bruit» [bryt] dans Las ordenanças ou encore d' "afachadas" (apprêtées) dans La Requeste face à "afaytadas» dans Las Ordenanças ${ }^{16}$. On peut ainsi voir dans la poussée des formes populaires orales à l'écrit que montrent ces textes du $\mathrm{XVI}^{\mathrm{e}}$ siècle l'effet de la ruine de la norme classique endogène, mise horsjeu par l'avènement et la socialisation d'une forme prestigieuse d'origine externe qui impose à son tour, par imitation, son fonctionnement sur la hiérarchisation des formes occitanes. La montée consciente, mais parfois aussi involontaire du bêtacisme dans ces textes, tout comme la disparition quasi généralisée de la plupart des anciennes formes prestigieuses en $\langle\mathrm{ch}\rangle$, peuvent être analysées comme des indices de la perte des contraintes sociolinguistiques internes de l'occitan.

C'est à présent un nouveau rapport conflictuel qui s'établit entre d'un côté une diglossie interne dans laquelle le beau langage toulousain s'oppose aux autres dialectes occitans - reproduisant ainsi le modèle du français face aux autres dialectes d'oïl, voire aux autres langues de France - et une diglossie externe entre occitan et français. La perte 
du caractère supradialectal de la graphie classique, qui a pu souvent être définie comme une koinè, et le choix d'une forme locale comme seule forme de référence écrite pourra aussi avoir comme conséquence d'exacerber les nationalismes locaux. L'idée que puisse exister un beau langage toulousain qui se place au-dessus des autres variétés dialectales et se permet de tourner en dérision les autres variétés dialectales a pu mener le poète Pèir de Garròs ( ca 1525-ca 1580), guidé à son tour par une forme de nationalisme gascon, à pousser plus loin encore et sans la moindre ambigüité le projet d'un beau langage gascon que la Requeste ne faisait qu'esquisser sur un mode parodique et ambigu pour le beau langage toulousain. Dans son adresse «Au lecteur » des Poesias gasconas $^{17}$, Garròs proclame sans ambigüité la supériorité esthétique du dialecte gascon qu'il place au-dessus de toutes les autres variétés : «Le langage specialement apelé Gascon, naturel a nous de Bearn, Comenge, d'Armagnac et autres, qui somes enclos entre les mons Pyrenees et la Garone, est beau pardessus les autres sés affins, et comme l'Attique entre les Grecz ${ }^{18}$ ». Si Pèir de Garròs, qui réadapte partiellement la norme endogène classique occitane au gascon met officiellement ses talents de poète au service de la Reine de Navarre, Jeanne d'Albret, à qui il dédie ses Psaumes ${ }^{19}$ et au prince de Navarre, futur Henri IV, dans ses Poesias gasconas, place la barre bien plus haut que les textes toulousains puisqu' il veut faire du gascon la langue littéraire officielle d'un royaume, celui de Navarre. La conséquence sera bien évidemment d'accentuer par le trait dialectal la rupture diatopique entre les dialectes occitans.

Pour rendre compte des spécificités phonétiques locales, les auteurs vont bientôt recourir systématiquement au code graphique exogène du français, utilisé comme un système phonétique. La graphie classique n'y résistera pas comme on le voit quelques années plus tard chez le poète toulousain Pèire Godolin (1580-1649) qui déclare : "Noü̈rigat de Toulouso, me play de manteni soun lengatge bèl, et capble de derrambulha touto sorto de councepcius» ${ }^{20}$ [Nourrisson de Toulouse, il me plaît

17. Pey De Garros, Poesias gasconas, Toulouse, Jacques Colomiez, 1567.

18. La citation est donnée dans son orthographe originale.

19. PEY DE Garros, Psaumes de David viratz en rhythme gascon, Toulouse, Jacques Moulinier, 1565.

20. Pierre Goudelin, Le Ramelet Moundi, Toulouse, Raymond Colomiès, 1617. 
de maintenir son beau langage, capable de démêler toutes sortes de concepts]. La progression de la diglossie est encore plus manifeste chez le poète provençal Loís Belaud de la Belaudiera (1543-1588) pour qui le choix de la graphie française pour transcrire l'occitan sera sans doute une façon de se rapprocher plus encore de la modernité littéraire française et de mieux articuler le passage d'une langue à l'autre dans ses recueils bilingues. Dans l'œuvre de Belaud le français est présent non seulement à travers le code graphique, mais il est déjà présent aussi comme métalangue, autrement dit comme langue du discours qui encadre la production en occitan. Chez lui, dédicaces, titres et commentaires des textes occitans sont systématiquement en français (Fin des rithmes Françoises, par L . D. la Bellaudière, Gentilhomme Prouvensal, Chançon de la cruauté de ma maistresse, Ode, sur la misère et pauvreté qu'on endure estant un homme ${ }^{21}$...). Choisir les conventions graphiques françaises pour faire entrer l'occitan dans cette modernité littéraire peut aussi être pour le poète provençal un choix délibéré à un moment où le modèle graphique de l'occitan médiéval peut sembler archaïque et dépassé, et renvoyer, tel le latin, à des usages pragmatiques ennuyeux, rigides ou trop éloignés de l'expressivité poétique, comme semble l'indiquer à la même époque et dans la même ville d'Aix, le provençal Jean de Nostredame qui dans son anthologie consacrée aux troubadours ${ }^{22}$, adapte la graphie des textes médiévaux selon les nouveaux codes graphiques francisés, semblant indiquer ainsi que la possibilité de décoder la graphie médiévale s'est perdue à jamais.

\section{Les débats graphiques avant la Renaissance du $\mathrm{XIX}^{\mathrm{e}}$ siècle}

Il faudra attendre le XVIII ${ }^{e}$ siècle pour que le débat autour des choix graphiques en occitan commence d'émerger à nouveau avec la publication de dictionnaires sur l'occitan. Que ces ouvrages fassent référence ou non à la tradition graphique médiévale, on y décèle deux tendances graphiques souvent antagonistes : un système de type phonétique tel qu'il s'est peu à peu installé depuis le $\mathrm{XVI}^{\mathrm{e}}$ siècle et

21. Sylvan CHABAUD (ed.), Louis Bellaud de la Bellaudière, Obros et Rimos (Sonnets et autres rimes de la prison), Montpellier, PULM, 2011.

22. Jean DE Nostredame, Les vies des plus célèbres et anciens Poètes provensaux, Lyon, pour A. Marsiii, 1575. 
qui tente de reproduire l'oralité au plus près ou un système de type "classique», faisant généralement référence à la tradition médiévale, qui laisse en partie de côté les réalisations orales dialectales.

\section{La conversion au français et à son système graphique}

C'est en Provence sans doute qu'apparaît un des premiers ouvrages lexicographiques d'éducation au français, le Dictionnaire Provençal \& François de Sauveur-André Pellas ${ }^{23}$, publié au début du XVIII ${ }^{\mathrm{e}}$ siècle. Par simple pragmatisme, l'auteur nous indique que son dictionnaire peut apparaître utile pour des «François» lorsqu' ils sont en Provence ${ }^{24}$, mais on retiendra toutefois que chez lui, il n'est pas question de défendre une quelconque dignité linguistique de l'occitan. D'une part la «langue provençale» n'est pas une langue, mais un mélange dénué de toute originalité linguistique "composé de la Françoise, de l'Italienne \& de l'Espagnole» ${ }^{25}$, d'autre part, la situation diglossique qui a consacré la primauté du français n'est absolument pas remise en cause. Comme il le dit sans l'expression du moindre état d'âme quant à la disparition possible de l'occitan, son ouvrage vise avant tout à convertir la population provençale à l'usage du français : «Comme aujourd'hui, \& depuis plus de deux siècles, la langue Françoise est devenuë la dominante en ce pays : c'est d'elle dont les Provençaux ont plus besoin, $\&$ pour l'acquisition de laquelle, aussi, cet ouvrage a été composé ${ }^{26} »$. Le français étant la langue-cible, il est acquis dans ce dictionnaire, comme dans bien d'autres qui suivront, que c'est dorénavant son code graphique qui servira aussi à représenter le lexique dit "patois». Donner une forme graphique différente ou autochtone au lexique occitan reviendrait à lui reconnaître une part d'originalité difficilement compatible avec la visée acculturatrice de l'ouvrage. Il va de soi que la dimension phonétique associée au code graphique exogène tend à accentuer les différences entre dialectes et contribue à l'impression de fragmentation linguistique de l'espace occitan. Ce type de productions sera abondant dans tout l'espace occitan jusqu'au XIX ${ }^{\mathrm{e}}$ siècle, à l'instar de l'Interprète provençal

23. Sauveur-André PELLAS, Dictionnaire Provençal et François, Avignon, chez François Sébastien Offray, 1723.

24. Ibidem, p. 4.

25. Ibid., p. 3.

26. Ibid., p. 4. 
dont l'auteur ne voit dans la langue qu'un système utilitaire commun de référence, dénué de tout héritage culturel et toute légitimité historique et qui lui permet d'affirmer que «la langue nationale devrait être seule en usage dans toute la France comme le sont aujourd'hui les nouveaux poids et mesures ${ }^{27}$.

On voit cependant se dessiner discrètement depuis le XVIII ${ }^{e}$ siècle une nouvelle approche plus positive de la langue occitane. Après avoir publié une première version de son dictionnaire ${ }^{28}$ accompagné d'un abrégé d'orthographe et de grammaire qui a pour seule vocation d'instruire les Provençaux et d'en faire de bons francophones, Étienne Garcin prétend dans la seconde édition de son dictionnaire vouloir «satisfaire» les Provençaux qui « soupirent après un Dictionnaire complet de leur idiome» ${ }^{29}$. Il change complètement le système graphique basé sur le français qu'il avait utilisé pour la première édition pour un système plus étymologique, inspiré des "productions de Raynouard et Diouloufet ». Entre le XVIII et le XIX ${ }^{\mathrm{e}}$ siècle, la redécouverte de l'occitan et des textes anciens a probablement conduit quelques lexicographes à modifier certains de leurs préjugés sur la langue et il semble ainsi que vers la seconde moitié du XIX ${ }^{e}$ siècle le conformisme linguistique autour de la promotion exclusive du français, qui semblait de rigueur particulièrement autour et après la Révolution, commence à faiblir et que s'ouvre un espace pour une nouvelle approche de l'occitan.

Le parcours de l'abbé Boissier de Sauvages est à ce titre particulièrement intéressant. La première édition de son dictionnaire en $1756^{30}$ affiche comme but principal d'être "un recueil des principales fautes que commettent dans la diction, $\&$ dans la Prononciation Françoise les Habitants des Provinces Méridionales du Royaume qui n'ont pas fait une étude particulière de cette langue». Cette perspective ne semblera

27. Jean-Jacques CASTOR, L'Interprète provençal, Apt, 1843, p. V-VI.

28. Étienne GARCIN, Le nouveau dictionnaire provençal-français, Marseille, Roche, 1823.

29. Étienne GARCIN, Le nouveau dictionnaire provençal-français, $2^{\mathrm{e}}$ édition augmentée, Draguignan, Fabre, 1841.

30. Pierre-Augustin BOISSIER DE SAUVAGES, Dictionnaire Languedocien-François, Nismes, M. Gaude, 1756. 
pas trop différente lors de la réédition du dictionnaire en $1785^{31}$. Cette deuxième édition contient cependant de véritables petits manifestes en faveur de l'occitan, une langue à laquelle il n'a manqué que de devenir la langue d'un pouvoir. Cette description est assez nouvelle à l'époque de Sauvages et se fonde sur l'idée d'une véritable cohérence linguistique au-delà des variétés dialectales. Pour cette langue qui «embrasse toute la partie méridionale de la Gaule», Sauvages donne une description géographique plus précise des limites linguistiques du domaine dans cette deuxième édition : "langage divisé autrefois, comme il continue de l'être aujourd'hui en différents dialectes; qui depuis Antibes jusqu'à Bordeaux, se rapprochent, se mêlent, se fondent, pour ainsi dire, par des nuances insensibles l'un dans l'autre ${ }^{32}$.» Cette édition comprend aussi un traité orthographique censé rendre compte de l'originalité de l'occitan, appelé «languedocien» par Sauvages. Toutefois son système graphique de type exogène qui intègre des digrammes de l'italien $(<\mathrm{gh}>$ par exemple), en propose aussi de nouveaux (comme $<\mathrm{jh}>$ ) et ajoute quantité indigeste de diacritiques sur les voyelles et diphtongues, repose sur des choix personnels peu compatibles avec une volonté de reconquête linguistique ${ }^{33}$. Si l'acceptabilité d'une norme se construit sur la reprise d'une tradition ou sur le recours à des pratiques familières, ce n'est pas le cas chez Sauvages dont les choix ont parfois de quoi surprendre ${ }^{34}$. Son seul but étant de rendre compte de sons absents du français, il fait fi de toute contrainte normative interne :

«Nous avons pris le parti de les copier, si l'on peut s'exprimer ainsi, de mot à mot \& d'écrire le Languedocien précisément comme nous le parlons. Nous n'étions génés [sic] par aucune des considérations qui doivent rendre un Auteur réservé lorsqu'il écrit dans une langue déjà assujettie à des règles, $\&$ dont l'orthographe est en quelque

31. Pierre-Augustin BOISSIER DE SAUVAGES, Dictionnaire Languedocien-François, Nismes, Gaude, 1785.

32. Ibidem, p. II.

33. Hervé LIEUTARD, «Le système graphique de l'abbé Boissier de Sauvages : défense et illustration de la diglossie franco-occitane", Lengas [En ligne], nº 68 | 2010, mis en ligne le 01 avril 2015, consulté le 16 décembre 2018. URL : http://journals. openedition.org/lengas/675; DOI : 10.4000/lengas.675.

34. Pour le son $[\mathrm{k}]$ par exemple, il utilise $<\mathrm{k}>$ («kichâdo») ou <q> («qan», «qatrë») en fonction du degré d'éloignement ou de proximité lexicale avec le français. 
sorte fixée, ou par les Grammairiens, ou par l'usage des bons écrivains $[. .$.$] Mais ces loix [s i c]$ ne sont point arbitraires; nous n'avons usé de notre droit que pour choisir entre les orthographes, celle qui nous a paru la plus simple $\&$ la plus facile, $\&$ nous nous flattons de l'avoir si bien assujettie à la prononciation, qu'elle en est une exacte et fidèle expression ${ }^{35}$.»

Tout en contribuant à multiplier les solutions graphiques personnelles et à entretenir la confusion sur la validité du système classique, le système graphique complexe que propose Sauvages a pour seul but de mettre en place un code qui soit au plus proche de la prononciation. Dans l'édition de 1785, il a du bien du mal à gérer la lemmatisation des nouvelles formes lexicales glanées dans des manuscrits médiévaux ou dans d'autres dialectes, étant incapable de voir une dimension autre que strictement orale dans les entrées qu'il propose. Cela dit, Sauvages pose comme définitive la rupture qualitative entre ce qu'il appelle la « langue d'oc ", autrement dit la langue médiévale et le « languedocien », dialecte qu'il limite à des fonctions linguistiques basses en usage dans la société occitane du XVIII ${ }^{e}$ siècle, mais qui n'a plus rien à voir avec la langue de prestige dans laquelle il puise son origine. Comme un certain nombre de lexicographes de son temps, il reste convaincu qu'il existe une variété orale "pure» d'occitan qui détermine sans doute ses choix graphiques et lexicaux : "passé ce terme, le son change, le françois disparoit, le gascon ${ }^{36}$ se développe, il devient insensiblement plus pur ; mais au-delà de ce dernier état, qui a quelque étendue, il dégénère et va se perdre également par nuances, d'un côté dans l'italien et de l'autre dans la langue espagnole.»

On voit dans ces quelques remarques que la disparition de la norme graphique endogène a effacé les contraintes qui pouvaient y être liées et que l'absence de toute autorité pouvant permettre de la faire vivre en a fait une quantité négligeable. Seul le français semble dorénavant capable de représenter une institution suffisamment solide pour pouvoir imposer une norme, pour lui-même, comme pour l'ensemble des langues qu'il domine.

35. P.-A. Boissier de SAuvages, op. cit., p. XVI.

36. Le terme «gascon» est pris ici dans le sens d'occitan. 


\section{Le retour de la graphie classique}

Dans le Dictionnaire de la Provence et du Comté Venaissin d'Achard paru en $1785^{37}$, la même année que la seconde édition du dictionnaire de Sauvages, le lexicographe provençal semble tout à fait décomplexé par rapport à une quelconque hiérarchie linguistique entre français et occitan. Il publie d'ailleurs quelque temps après la sortie du premier tome contenant le Vocabulaire Provençal-François, un second tome contenant le Vocabulaire François-Provençal, dont le but clairement exprimé est de faciliter l'accès au provençal à ceux qui ne le parlent pas. Achard n'hésite pas à rejeter en bloc la graphie phonétique : «Tous les auteurs modernes qui ont écrit en provençal se sont fait une orthographe arbitraire ; le plus grand nombre a écrit le Provençal comme on le parle ${ }^{38}$ ". Il va donc utiliser des formes plus proches de l'étymologie et de la langue ancienne : «Nous avons cru devoir établir une Sintaxe [sic] provençale qui se rapprochant de l'ancienne, facilitera aux Provençaux la lecture des Écrivains en cette langue qui viendront après nous, ou qui nous ont précédés ${ }^{39} »$. Achard comprend qu'une graphie de type classique est mieux à même de rendre compte de la variation diachronique. Mieux encore, il conçoit aussi la nécessité de rendre compte de la variation diatopique à travers ses choix graphiques, non pas en multipliant les entrées de son dictionnaire comme le fait Sauvages, mais en faisant le choix de formes englobantes. Il justifie aussi le choix de la notation graphique du $<s>$ du pluriel, amuï dans le provençal maritime et rhodanien, par une approche à la fois diachronique et synchronique de la langue ${ }^{40}$. Il n'est donc pas question pour lui de mettre en place un sous-système graphique du français et la distance entre graphie et phonie qu'il préconise trouve au-delà de sa fonction de distinction des formes homophones une réelle justification dans une observation des réalisations dialectales qui manifeste un respect des variétés septentrionales que d'autres méprisent à la même époque :

37. Claude-François ACHARD, Dictionnaire de la Provence et du Comté Venaissin. Tome Premier contenant le vocabulaire François-Provençal. Tome second, contenant le vocabulaire Provençal-François, Marseille, Imprimerie Jean Mossy, 1785.

38. Ibidem, p. XII.

39. Ibid., p. XII.

40. Ibid., p. XIV. 
«Nous avons distingué les Infinitifs des verbes par une $\mathrm{R}$ finale. Les Participes \& les Adjectifs masculins se terminent par un T. Nous écrivons acabar, achever, \& acabat, achevé, quoique nous prononcions acaba dans l'un \& l'autre sens. Nous sentons bien que cette orthographe doit gêner les Poëtes provençaux, mais c'est la plus naturelle \& les Provençaux qui habitent dans les montagnes ont conservé la prononciation finale des lettres $\mathrm{R}$ et $\mathrm{T}$, preuve certaine qu' elles existent dans notre langue ${ }^{41}$.»

En puisant dans les ressources historiques et en observant de près les réalisations dialectales, Achard résout la plupart des questions graphiques insolubles que se pose sans doute Sauvages au même moment, même si pour Achard, le terme "provençal " n'est pas comparable à la définition élargie à l'espace occitan qu'en donnera plus tard Mistral dans le Tresor dóu Felibrige ${ }^{42}$ ni au terme de "languedocien" qu'utilise Sauvages dans le sens de langue d'oc moderne, puisque c'est le seul dialecte provençal qu'Achard érige en langue, nettement distincte des autres variétés de la langue d'oc.

Le XIX ${ }^{e}$ siècle, notamment grâce à la relecture des troubadours, représente une prise de conscience nouvelle des liens génétiques qui peuvent unir langue populaire et langue médiévale. Le courant classiciste naissant, dont la Provence apparaît comme la figure de proue depuis la parution du dictionnaire d'Achard, étend les usages graphiques médiévaux à la langue moderne pour mieux souligner la continuité diachronique et la cohérence diatopique des usages. C'est d'ailleurs ce que fera Honnorat, médecin de Digne, qui dans la première moitié $\mathrm{du} \mathrm{XIX}^{\mathrm{e}}$ siècle, fait preuve dans son dictionnaire ${ }^{43}$ d'une plus grande maîtrise de la linguistique et de la lexicographie qu'un grand nombre de ces prédécesseurs ${ }^{44}$. Pleinement conscient de l'influence du français sur l'occitan, il est à même de proposer dans son dictionnaire des

\section{Ibid., p. XVII.}

42. Frédéric Mistral, Lou tresor dóu Felibrige, Aix, Edisud, 1882-1886 (Reprint 1979).

43. Simon-Jude HonnOrat, Dictionnaire Provencal-Français ou dictionnaire de la langue d'Oc ancienne et moderne, Digne, Repos éditeur, 1846-1847.

44. Philippe MARTEL, "Du parler local à la langue : le Docteur Honnorat à la découverte de l'unité de la langue d'oc», Chroniques de Haute-Provence, n 365, p. 3466, Société Scientifique et Littéraire des Alpes de Haute-Provence, 2010. 
solutions pour rejeter les formes diglossiques, mais surtout de proposer un système graphique unitaire susceptible de recouvrir l'ensemble des usages. Il favorise dans son dictionnaire le recours à l'étymologie pour le choix des entrées, mettant ainsi au second plan son propre dialecte vivaro-alpin de Digne ("Madur > maur», mûr). C'est toutefois côté languedocien sans doute à Rochegude ${ }^{45}$ que l'on doit de démontrer avec le plus de constance la continuité entre l'occitan médiéval et l'occitan moderne. Il est le premier promoteur de l'adjectif «occitanien" qui envisage la langue dans toute sa dimension diachronique et dans toute son étendue géographique. Le fait de placer l'occitan sur le même plan que les autres langues romanes dans la préface de son ouvrage est déjà une façon de lui rendre une dignité linguistique perdue : «Le français supplanta l'occitanien, qui malgré ses avantages fut entièrement délaissé : on l'a même depuis injustement relegué dans la classe des patois. Cependant des millions de personnes parlent encore cette langue ${ }^{46} »$. Rochegude va même jusqu'à préciser la valeur phonétique des graphèmes et digrammes de la langue écrite médiévale en se servant notamment de la prononciation languedocienne contemporaine telle que le bêtacisme pour expliquer la façon dont les textes anciens doivent être lus ( $\mathrm{V}$ se prononce comme $\mathrm{B} »{ }^{47}$ ). Il s'écarte clairement de l'usage des graphies phonétiques, notamment en transcrivant les diphtongues selon la tradition romane $<$ au $>,<a i>$, etc. ou en reprenant les digrammes médiévaux autochtones $<\mathrm{lh}>$ et $<\mathrm{nh}>$. Cette conception d'un continuum diachronique de la langue occitane est complétée par une vision nouvelle, ébauchée toutefois chez Sauvages, d'une continuum diatopique de la langue qui n'est plus compatible avec le terme de "patois» ni avec une multiplication de solutions graphiques hybrides.

Dès la dédicace de La langue d'oc rétablie, le languedocien Fabre d'Olivet ${ }^{48}$ affirme lui aussi cette conception d'une continuitélinguistique occitane «des Alpes aux Pyrénées », expression que reprendra Mistral ${ }^{49}$.

45. Henri Pascal DE Rochegude, Essai d'un glossaire occitanien, pour servir à l'intelligence des poésies des troubadours, Toulouse, Benichet-Cadet, 1819.

46. Ibidem, p. XXXIX.

47. Ibid., p. XLVIII-XLIX.

48. Antoine FABRE D'OLIVET, La langue d'oc rétablie (première édition intégrale d'après le manuscrit de 1820), Barcelone, Association Fabre d'Olivet, 1989.

49. Frédéric Mistral, Lis Isclo d'or, Paris, Alphonse Lemerre, 1876. 
Il rejette «le nom ignoble de patois ${ }^{50}$ pour l'adjectif «occitanique» et propose un nouveau système graphique qui doit pouvoir montrer le lien qui unit l'occitan aux autres langues romanes, notamment en rétablissant la voyelle $<$ a $>$ post-tonique, souvent notée $<$ o $>$ parce qu'elle est majoritairement prononcée ainsi : "Cette coïncidence entre les trois langues méridionales, l'espagnole, l'oscitanique et l'italienne, doit convaincre d'erreur ceux qui s'attachent à un dialecte fautif, s'obstinent à substituer l'o à l'a dans les désinences féminines ${ }^{51}$. En dernier lieu, à la fin du troisième chapitre de sa Grammaire, Fabre d'Olivet illustre ses choix graphiques en transposant dans son système des extraits d'auteurs occitans qui ont utilisé le système exogène français, comme par exemple de Godolin à Toulouse au XVII ${ }^{e}$ siècle. À travers cet exercice, il s'agit pour Fabre d'Olivet de montrer la continuité linguistique entre l'occitan des troubadours et celui du poète toulousain : "Il est facile de voir, en parcourant les divers morceaux que je viens de donner, qu'ils appartiennent tous au même idiome, et qu'ils ne diffèrent entre eux que par quelques changements que le temps a dû nécessairement apporter ${ }^{52} »$. Fabre d'Olivet fait ici une démonstration sans doute plus parlante que tous les discours sur la graphie. En transposant les textes des siècles précédents vers un système plus classique, il nous donne finalement l'image graphique de ce que ces textes auraient dû être ou auraient pu être si la tradition classique n'avait pas été victime d'une rupture diglossique. Il est évident que pour Fabre d'Olivet les choix graphiques posent la dignité de la langue. Plus encore, ils permettent de rétablir la continuité linguistique que la diglossie a occultée, notamment à travers la francisation du système graphique.

\section{Conclusion}

L'avènement du Félibrige en 1854 sonnera brutalement le glas des tentatives restauratrices de type classique en Provence et dans le reste de l'espace occitan. C'est d'ailleurs en paraphrasant Sauvages que Roumanille, inventeur et promoteur de la graphie du Félibrige ${ }^{53}$

50. A. FABRE D'OliVET, op. cit., p. 640.

51. Ibidem, p. 56.

52. A. FABRE D'OliveT, op. cit., p. 47.

53. Joseph Roumanille, La part dau boun Diéu, Avignon, Séguin ainé, 1853. 
dira que la «règle fondamentale, c'est que la prononciation est dans notre langue, le guide de l'orthographe [...] ». Si Mistral s'oppose dans un premier temps à Roumanille sur la question graphique, la volonté de ne pas renoncer à des particularités phonétiques considérées comme une dimension essentielle de l'expressivité poétique de Provence conduira finalement le Félibrige au choix d'une graphie phonétique. Même si elle reprend le système de transcription des diphtongues de l'écrit médiéval et tente de renouer avec le prestige littéraire des troubadours, la norme félibréenne effectue consciemment une rupture avec l'héritage graphique médiéval en intégrant une partie des conventions graphiques françaises. Dans tous les cas, la volonté de Roumanille d'illustrer par ses choix graphiques le «caractère si coulant, si mielleux, si ionien » du provençal rhodanien tout en rejetant le " caractère si rude, si âpre, si déplaisant à l'oreille ${ }^{54}$ de la variété occitane alpine - allusion à peine déguisée à Honnorat et à son dictionnaire - ira à l'encontre du principe d'unité de la langue d'oc ou du moins se montrera incompatible avec l'idée même d'une forme écrite supradialectale d'occitan qui passerait par l'usage de la graphie classique. Si le Félibrige se veut au départ un mouvement poétique dont la portée se réduit aux environs d'Avignon, ses choix graphiques originels apparaîtront par la suite en totale inadéquation avec le projet d'un mouvement littéraire à l'échelle de tout l'espace occitan, que ce soit chez des Félibres limousins tels que Joseph Roux qui renouera avec le modèle graphique des troubadours limousins, ou encore dans les premières dissidences languedociennes qui ne manqueront pas de se produire quelques années après l'institutionnalisation du Félibrige ${ }^{55}$. Il aura sans doute manqué à ce moment-là au Félibrige une capacité suffisante de remise en question et de réforme de ses choix orthographiques initiaux. Il faudra attendre le début du XXe siècle pour que, côté languedocien, l'usage de la graphie occitane dite classique, propose un nouveau système englobant, susceptible de gérer la variation dialectale tout en se reconnectant aux usages médiévaux. Ce système,

54. Ibidem, p. 34.

55. Philippe MARTEL, «Une norme pour la langue d'oc ? Les débuts d'une histoire sans fin", Lengas [En ligne], $\mathrm{n}^{\circ} 72$ |2012, mis en ligne le 25 octobre 2013, consulté le 14 janvier 2019. URL : http://journals.openedition.org/lengas/112; DOI : 10.4000/ lengas. 112. 
notamment après la parution de la Gramatica de Loís Alibèrt ${ }^{56}$ et sa diffusion par l'IEO, s'est progressivement répandu à tout l'espace occitan au cours du XXe siècle en réduisant l'usage de la graphie mistralienne. Au-delà de sa coexistence pacifique avec la graphie mistralienne aujourd'hui en Provence, sa progression a toutefois mis au jour des dissensions importantes, souvent très anciennes, qui montrent que tout système graphique, au-delà de sa simple fonction de transcription d'une langue à l'écrit, est aussi le véhicule de toutes les représentations liées à la langue.

56. Loís ALIBÈRT, Gramatica occitana segon los parlars lengadocians, Tolosa, Societat d'Estudis Occitan, 1935. 